Journal of Applied AnALysis

Vol. 13, No. 1 (2007), pp. 19-31

\title{
ON A JENSEN TYPE FUNCTIONAL EQUATION
}

\author{
W. SMAJDOR
}

Received February 24, 2005 and, in revised form, February 6, 2006

\begin{abstract}
We investigate the functional equation

$$
\begin{aligned}
& \text { af } \frac{x+y+z}{3}+f(x)+f(y)+f(z) \\
& =b\left[f \frac{x+y}{2}+f \frac{y+z}{2}+f \frac{z+x}{2}\right]
\end{aligned}
$$

for $f: M \rightarrow S$, where $M$ is an Abelian 2- and 3-divisible group and $S$ is an abstract convex cone. The motivation for studying this equation came from results due to Tiberiu Trief [8] and Young Whan Lee [3], where equation (1) was considered with constants $a=3, b=2$ and $a=9$ and $b=4$, respectively.
\end{abstract}

1. Let $(M,+)$ be an Abelian group in which the unique division by 2 and 3 is performable. Let $(S,+)$ be an Abelian semigroup. Suppose that $S$ contains the identity element 0 and for each $\lambda \geq 0$ and $s \in S$, an element $\lambda \cdot s$ in $S$ is defined. It is assumed that the multiplication $[0, \infty) \times S \ni(\lambda, s) \longmapsto \lambda \cdot s \in S$ satisfies the following axioms:

$$
\begin{array}{ll}
1 \cdot s=s, & \lambda(\mu \cdot s)=(\lambda \mu) \cdot s, \\
\lambda \cdot(s+t)=\lambda \cdot s+\lambda \cdot t, & (\lambda+\mu) \cdot s=\lambda \cdot s+\mu \cdot s
\end{array}
$$

2000 Mathematics Subject Classification. Primary: 39B52, 39B82.

Key words and phrases. Jensen's functional equation, abstract convex cone, additive and quadratic functions, multifunctions.

ISSN 1425-6908（C) Heldermann Verlag. 
for all $s, t \in S$ and $\lambda, \mu \geq 0$. Then $S$ is said to be an abstract convex cone (see e.g. [7]).

If $s, t, t^{\prime} \in S, t+s=t^{\prime}+s$ always implies that $t=t^{\prime}$, then $S$ is said to satisfy the cancellation law.

Suppose that an invariant with respect to translations and positively homogeneous metric $\varrho$ is given in $S$, i.e.,

$$
\varrho\left(t+s, t^{\prime}+s\right)=\varrho\left(t, t^{\prime}\right)
$$

and

$$
\varrho(\lambda s, \lambda t)=\lambda \varrho(s, t)
$$

for $\lambda>0$ and $s, t, t^{\prime} \in S$.

It is easy to see that the mappings $[0, \infty) \times S \ni(\lambda, s) \longmapsto \lambda \cdot s \in S$ and $S \times S \ni(s, t) \longmapsto s+t \in S$ are continuous in the metric topology.

We are going to examine functional equation (1) where $a, b$ are nonnegative constants and $f$ is an unknown function defined in $M$ with values in $S$. Equation (1) in the case $a=3, b=2$ was studied in the paper of Tiberiu Trif [8] in the class of functions $f: X \rightarrow Y$, where $X$ and $Y$ are real vector spaces. For the same $a$ and $b$ equation (1) was considered in [6] for functions $f: M \rightarrow S$. In paper [6] it has been shown that every solution of the equation

$$
\begin{aligned}
& 3 f\left(\frac{x+y+z}{3}\right)+f(x)+f(y)+f(z)= \\
& 2\left[f\left(\frac{x+y}{2}\right)+f\left(\frac{y+z}{2}\right)+f\left(\frac{z+x}{2}\right)\right]
\end{aligned}
$$

has to be of the form

$$
f(x)=f(0)+a(x),
$$

where $a: M \rightarrow S$ is an additive function. In the case $a=9$ and $b=4$ equation (1) was considered in paper [3] of Yong Whan Lee also in the class of functions $f: X \rightarrow Y$, where $X, Y$ are real vector spaces.

One could believe that the natural domain of equation (2) is a convex set. The following example shows that there are solutions $f: M \rightarrow S$ of (2) which does not have to be of form (3). We take $f(x)=[0,1-x]$ for $x \in[0,1]$. This function has values in the convex cone $c c(\mathbb{R})$ of all non-empty convex compact subsets of $\mathbb{R}$ and is a solution of the Jensen functional equation

$$
f\left(\frac{x+y}{2}\right)=\frac{1}{2}(f(x)+f(y))
$$

so it is a solution of (2) but it cannot be represented in the form $f(x)=$ $f(0)+a(x)$, where $a$ is an additive function (cf. [5]). 
2. In the sequel we will assume that

(a) $M$ is an Abelian group with zero in which the unique division by 2 and 3 is performable;

(b) $S$ is an abstract cone satisfying the cancellation law;

(c) $(S, \rho)$ is a complete metric space and $\rho$ is translation invariant and positively homogeneous.

We start from the following lemma.

Lemma 1. If $\lambda \cdot s=\mu \cdot s$ for some $\lambda, \mu \geq 0$ and $s \in S$, then $s=0$ or $\lambda=\mu$.

Proof. If $\lambda \neq \mu$, for example $\lambda>\mu$, then $\lambda \cdot s=((\lambda-\mu)+\mu) \cdot s=(\lambda-\mu)$. $s+\mu \cdot s$, whence $(\lambda-\mu) \cdot s=0$. Thus $0=\rho((\lambda-\mu) \cdot s, 0)=(\lambda-\mu) \rho(s, 0)$. Since $\lambda \neq \mu, \rho(s, 0)=0$ and $s=0$ follows.

Of course, the zero function is a solution of (1).

Proposition. If $f: M \rightarrow S$ is a non-zero solution of (1), then

$$
a=3(b-1) .
$$

Proof. There exists an $x_{0} \in M$ such that $f\left(x_{0}\right) \neq 0$. Setting $x=y=z$ $=x_{0}$ in (1) we obtain

$$
(a+3) f\left(x_{0}\right)=3 b f\left(x_{0}\right) .
$$

Thus by Lemma 1 formula (5) follows.

Since $a, b$ are non-negative constants, $b \geq 1$.

Theorem 1. Let $a=3(b-1)$. If $f: M \rightarrow S$ is a solution of (4), then $f$ satisfies (1). Conversely, if $b \geq 1, b \neq 4$, and $f: M \rightarrow S$ is a solution of (1), then $f$ satisfies (4).

Proof. Suppose that $f: M \rightarrow S$ is a solution of (4). Since $f((1 / 3)(x+y+z))=(1 / 3)(f(x)+f(y)+f(z))$,

$$
3(b-1) f\left(\frac{x+y+z}{3}\right)+f(x)+f(y)+f(z)=b[f(x)+f(y)+f(z)] .
$$

The right hand side of (1), in virtue of (4), is also equal to $b[f(x)+f(y)+f(z)]$, so the first statement of the theorem follows.

Now, assume that $b=1$ and that $f$ is a solution of (1). Then $a=0$ and $f$ satisfies the equation

$$
f(x)+f(y)+f(z)=f\left(\frac{x+y}{2}\right)+f\left(\frac{y+z}{2}\right)+f\left(\frac{z+x}{2}\right) .
$$


By letting $y=z=0$ in (6), we infer

$$
f(x)+f(0)=2 f\left(\frac{x}{2}\right) .
$$

Next putting $z=0$ in (6) we obtain hence

$$
\begin{aligned}
& f(x)+f(y)+f(0)=f\left(\frac{x+y}{2}\right)+f\left(\frac{x}{2}\right)+f\left(\frac{y}{2}\right) \\
& =f\left(\frac{x+y}{2}\right)+\frac{1}{2} f(x)+\frac{1}{2} f(0)+\frac{1}{2} f(y)+\frac{1}{2} f(0) \\
& =f\left(\frac{x+y}{2}\right)+\frac{1}{2} f(x)+\frac{1}{2} f(y)+f(0) .
\end{aligned}
$$

Consequently $f$ satisfies (4).

Now suppose that $b>1$. Let us assume that $f$ is a solution of (1) and write

$$
g(x):=\frac{1}{2}(f(x)+f(-x)), \quad x \in M .
$$

Of course $g$ is a solution of (1), i.e.,

$$
\begin{aligned}
& 3(b-1) g\left(\frac{x+y+z}{3}\right)+g(x)+g(y)+g(z) \\
& =b\left[g\left(\frac{x+y}{2}\right)+g\left(\frac{y+z}{2}\right)+g\left(\frac{z+x}{2}\right)\right],
\end{aligned}
$$

$g$ is even and $g(0)=f(0)$.

We note that $x=y+z=0$ in (7) gives

$$
3(b-1) g(0)+g(0)+2 g(y)=2 b g\left(\frac{y}{2}\right)+b g(0) \text {. }
$$

Hence

$$
2(b-1) g(0)+b g(0)+2 g(y)=2 b g\left(\frac{y}{2}\right)+b g(0)
$$

and after cancelling $b g(0)$ we have

$$
(b-1) g(0)+g(y)=b g\left(\frac{y}{2}\right),
$$

whence

$$
g(y)=\frac{1}{b} g(2 y)+\left(1-\frac{1}{b}\right) g(0) .
$$

Inserting $2 y$ instead of $y$ we get

$$
g(2 y)=\frac{1}{b} g\left(2^{2} y\right)+\left(1-\frac{1}{b}\right) g(0) .
$$


We substitute the last equality into (8) to obtain

$$
g(y)=\frac{1}{b^{2}} g\left(2^{2} y\right)+\left(1-\frac{1}{b^{2}}\right) g(0) .
$$

By induction

$$
g(y)=\frac{1}{b^{n}} g\left(2^{n} y\right)+\left(1-\frac{1}{b^{n}}\right) g(0)
$$

for all $y \in M$ and $n \in \mathbb{N}$.

We will prove that the sequence $\left(\left(1 / b^{n}\right) g\left(2^{n} y\right)\right)$ satisfies the Cauchy condition. For every positive integers $m$ and $n$ we have by (9)

$$
\begin{aligned}
& \varrho\left(\frac{1}{b^{m+n}} g\left(2^{m+n} y\right), \frac{1}{b^{n}} g\left(2^{n} y\right)\right) \\
& =\varrho\left(\frac{1}{b^{m+n}} g\left(2^{m+n} y\right)+\left(1-\frac{1}{b^{m+n}}\right) g(0), \frac{1}{b^{n}} g\left(2^{n} y\right)+\left(1-\frac{1}{b^{n}}\right) g(0)\right. \\
& \left.+\left(\frac{1}{b^{n}}-\frac{1}{b^{m+n}}\right) g(0)\right)=\varrho\left(g(y), g(y)+\left(\frac{1}{b^{n}}-\frac{1}{b^{m+n}}\right) g(0)\right) \\
& =\varrho\left(0,\left(\frac{1}{b^{n}}-\frac{1}{b^{m+n}}\right) g(0)\right)=\left(\frac{1}{b^{n}}-\frac{1}{b^{m+n}}\right) \varrho(0, g(0))
\end{aligned}
$$

Thus we may define the function $G: M \rightarrow S$ as follows

$$
G(y)=\lim _{n \rightarrow \infty} \frac{1}{b^{n}} g\left(2^{n} y\right) .
$$

Of course $G(0)=0, G$ is also even and by (9)

$$
g(y)=g(0)+G(y)=f(0)+G(y), \quad y \in M .
$$

Setting $2^{n} x, 2^{n} y, 2^{n} z$ instead of $x, y, z$, respectively, in (7) we obtain on letting $n \rightarrow \infty$,

$$
\begin{aligned}
& 3(b-1) G\left(\frac{x+y+z}{3}\right)+G(x)+G(y)+G(z) \\
& =b\left[G\left(\frac{x+y}{2}\right)+G\left(\frac{y+z}{2}\right)+G\left(\frac{z+x}{2}\right)\right],
\end{aligned}
$$

i.e., $G$ is a solution of (1). By (10)

$$
G(2 y)=\lim _{n \rightarrow \infty} \frac{1}{b^{n}} g\left(2^{n+1} y\right)=b \lim _{n \rightarrow \infty} \frac{1}{b^{n+1}} g\left(2^{n+1} y\right)=b G(y) .
$$

Thus (12) becomes

$$
\begin{aligned}
& 3(b-1) G\left(\frac{x+y+z}{3}\right)+G(x)+G(y)+G(z) \\
& =G(x+y)+G(y+z)+G(z+x) .
\end{aligned}
$$


If we put $y=z=0$, then we obtain the condition

$$
3(b-1) G\left(\frac{x}{3}\right)=G(x), \quad x \in M .
$$

This equality and (13) lead to

$$
G(x+y+z)+G(x)+G(y)+G(z)=G(x+y)+G(y+z)+G(z+x) .
$$

Taking $y+z=0$ we obtain

$$
2 G(x)+2 G(y)=G(x+y)+G(x-y), \quad x, y \in M,
$$

so $G$ is a quadratic function. By (14)

$$
3(b-1) G(x)=G(3 x), \quad x \in M .
$$

On the other hand $G(3 x)=9 G(x)$ for each $x \in M$. Thus $3 G(x)=$ $(b-1) G(x), x \in M$. Note that by the assumption $b \neq 4$. Consequently by Lemma $1, G=0$ in $M$. So by (11) we get $g(y)=f(0)$ for $y \in M$, whence $f(x)+f(-x)=2 f(0)$ for all $x \in M$.

Putting $z=-y$ in (1) we obtain

$3(b-1) f\left(\frac{x}{3}\right)+f(x)+f(y)+f(-y)=b\left[f\left(\frac{x+y}{2}\right)+f(0)+f\left(\frac{x-y}{2}\right)\right]$.

Hence

$$
3(b-1) f\left(\frac{x}{3}\right)+f(x)+2 f(0)=b\left[f\left(\frac{x+y}{2}\right)+f\left(\frac{x-y}{2}\right)\right]+b f(0) .
$$

We observe that the left-hand side does not depend on $y$. So setting $y=0$ in the above equality and comparing the right-hand sides we derive

$$
f\left(\frac{x+y}{2}\right)+f\left(\frac{x-y}{2}\right)=2 f\left(\frac{x}{2}\right), \quad x, y \in M .
$$

Consequently $f$ is a solution of the Jensen functional equation (4).

It is not difficult to check that every quadratic function $q: M \rightarrow S$ is a solution of the equation

$$
\begin{aligned}
& 9 f\left(\frac{x+y+z}{3}\right)+f(x)+f(y)+f(z) \\
& =4\left[f\left(\frac{x+y}{2}\right)+f\left(\frac{y+z}{2}\right)+f\left(\frac{z+x}{2}\right)\right] .
\end{aligned}
$$

Analysing the proof of Theorem 1 one may obtain the following result.

Theorem 2. If $f: M \rightarrow S$ is a function, then $f$ is an even solution of (15) if and only if there exists a quadratic function $q: M \rightarrow S$ such that

$$
f(x)=f(0)+q(x), \quad x \in M .
$$


3. We do not know if every solution $f: M \rightarrow S$ of (15) has to be of the form $f(x)=f(0)+a(x)+q(x), x \in M$, where $a: M \rightarrow S$ is an additive function and $q: M \rightarrow S$ is a quadratic one. To obtain more informations about solutions of equation (15) we will embed the abstract convex cone $S$ into a real vector space. We use the idea of H. Rådström (cf. [4]).

The equivalence relation in $S \times S=S^{2}$ is defined as follows

$$
(s, t) \sim(u, v) \Leftrightarrow s+v=t+u .
$$

The equvalence class containing a pair $(s, t)$ is denoted by $[s, t]$. The quotient space $S^{2} / \sim$ is denoted by $X$. We define the addition in $X$ by the formula

$$
[s, t]+[u, v]=[s+u, t+v]
$$

and if $\lambda \geq 0$, then

while if $\lambda<0$, then

$$
\lambda[s, t]=[\lambda \cdot s, \lambda \cdot t]
$$

$$
\lambda[s, t]=[-\lambda \cdot t,-\lambda \cdot s] .
$$

With these operations the set $X$ becomes a real vector space.

Suppose that $f: M \rightarrow S$ is a solution of (15). It is easy to check that the function $F: M \rightarrow X$

$$
F(x):=[f(x), f(0)]
$$

is a solution of the equation

$$
\begin{aligned}
& 9 F\left(\frac{x+y+z}{3}\right)+F(x)+F(y)+F(z) \\
& =4\left[F\left(\frac{x+y}{2}\right)+F\left(\frac{y+z}{2}\right)+F\left(\frac{z+x}{2}\right)\right] .
\end{aligned}
$$

Conversely, if $F$ given by (16) satisfies (17), then $f$ is a solution of (15).

Theorem 3. If $f: M \rightarrow S$ is a solution of (15), then functions $a, q: M \rightarrow$ $X$ defined as follows

$$
\begin{aligned}
& a(x)=\frac{1}{2}[f(x), f(-x)], \\
& q(x)=\frac{1}{2}[f(x)+f(-x), 2 f(0)]
\end{aligned}
$$

satisfy equation (17) and $a$ is an additive function, $q$ is a quadratic one. Moreover $F(x)=a(x)+q(x), x \in M$, where $F$ is given by formula (16).

Proof. We observe that $a(0)=0$ and $q(0)=0$, where the second zero in the last equalities is zero of the vector space $X$. Further,

$$
a(-x)=\frac{1}{2}[f(-x), f(x)]=-\frac{1}{2}[f(x), f(-x)]=-a(x)
$$


and

$$
q(-x)=\frac{1}{2}[f(x)+f(-x), 2 f(0)]=q(x) .
$$

Now we are going to show that $a$ is a solution of (17). By the definition of operations "+" and "." we have

$$
\begin{aligned}
& 18 a\left(\frac{x+y+z}{3}\right)+2 a(x)+2 a(y)+2 a(z) \\
& =9\left[f\left(\frac{x+y+z}{3}\right), f\left(-\frac{x+y+z}{3}\right)\right] \\
& +[f(x), f(-x)]+[f(y), f(-y)]+[f(z), f(-z)] \\
& =\left[9 f\left(\frac{x+y+z}{3}\right)+f(x)+f(y)+f(z),\right. \\
& \left.9 f\left(-\frac{x+y+z}{3}\right)+f(-x)+f(-y)+f(-z)\right] \\
& =4\left[f\left(\frac{x+y}{2}\right)+f\left(\frac{y+z}{2}\right)+f\left(\frac{z+x}{2}\right),\right. \\
& \left.f\left(-\frac{x+y}{2}\right)+f\left(-\frac{y+z}{2}\right)+f\left(-\frac{z+x}{2}\right)\right] \\
& =4\left[f\left(\frac{x+y}{2}\right), f\left(-\frac{x+y}{2}\right)\right] \\
& +4\left[f\left(\frac{y+z}{2}\right), f\left(-\frac{y+z}{2}\right)\right]+4\left[f\left(\frac{z+x}{2}\right), f\left(-\frac{z+x}{2}\right)\right] \\
& =8 a\left(\frac{x+y}{2}\right)+8 a\left(\frac{y+z}{2}\right)+8 a\left(\frac{z+x}{2}\right) .
\end{aligned}
$$

Hence

$$
\begin{aligned}
& 9 a\left(\frac{x+y+z}{3}\right)+a(x)+a(y)+a(z) \\
& =4 a\left(\frac{x+y}{2}\right)+4 a\left(\frac{y+z}{2}\right)+4 a\left(\frac{z+x}{2}\right) .
\end{aligned}
$$

In order to prove that $a$ is additive at first we put $y+z=0$ in (18). Then

$$
9 a\left(\frac{x}{3}\right)+a(x)=4 a\left(\frac{x+y}{2}\right)+4 a\left(\frac{x-y}{2}\right) .
$$

Next, putting $y=x$ in (19) we have

$$
9 a\left(\frac{x}{3}\right)=3 a(x) .
$$

Thus

$$
a(3 x)=3 a(x), \quad x \in M
$$


Further letting $x=3 y$ in (19) we get

$$
9 a(y)+a(3 y)=4 a(2 y)+4 a(y) .
$$

This equality and (20) imply

$$
a(2 y)=2 a(y), \quad y \in M .
$$

From (18) taking into account (20) and (21) we obtain

$$
3 a(x+y+z)+a(x)+a(y)+a(z)=2 a(x+y)+2 a(y+z)+2 a(z+x) .
$$

For $z=-x-y$ we have hence

$$
a(x)+a(y)-a(x+y)=2 a(x+y)-2 a(x)-2 a(y),
$$

whence the additivity of $a$ follows.

Now we will show that $q$ is also a solution of (17). To see this note that

$$
\begin{aligned}
& 18 q\left(\frac{x+y+z}{3}\right)+2 q(x)+2 q(y)+2 q(z) \\
& =9\left[f\left(\frac{x+y+z}{3}\right)+f\left(-\frac{x+y+z}{3}\right), 2 f(0)\right] \\
& +[f(x)+f(-x), 2 f(0)]+[f(y)+f(-y), 2 f(0)]+[f(z)+f(-z), 2 f(0)] \\
& =\left[9 f\left(\frac{x+y+z}{3}\right)+f(x)+f(y)+f(z)+9 f\left(-\frac{x+y+z}{3}\right)\right. \\
& +f(-x)+f(-y)+f(-z), 24 f(0)] \\
& =\left[4 f\left(\frac{x+y}{2}\right)+4 f\left(\frac{y+z}{2}\right)+4 f\left(\frac{z+x}{2}\right)\right. \\
& \left.+4 f\left(-\frac{x+y}{2}\right)+4 f\left(-\frac{y+z}{2}\right)+4 f\left(-\frac{z+x}{2}\right), 24 f(0)\right] \\
& =4\left[f\left(\frac{x+y}{2}\right)+f\left(-\frac{x+y}{2}\right), 2 f(0)\right] \\
& +4\left[f\left(\frac{y+z}{2}\right)+f\left(-\frac{y+z}{2}\right), 2 f(0)\right] \\
& +4\left[f\left(\frac{z+x}{2}\right)+f\left(-\frac{z+x}{2}\right), 2 f(0)\right] \\
& =8 q\left(\frac{x+y}{2}\right)+8 q\left(\frac{y+z}{2}\right)+8 q\left(\frac{z+x}{2}\right) .
\end{aligned}
$$

Thus $q$ satisfies the functional equation

$$
\begin{aligned}
& 9 q\left(\frac{x+y+z}{3}\right)+q(x)+q(y)+q(z) \\
& =4 q\left(\frac{x+y}{2}\right)+4 q\left(\frac{y+z}{2}\right)+4 q\left(\frac{z+x}{2}\right) .
\end{aligned}
$$


Now we proceed to show that $q$ is quadratic. We substitute $x+z=0$ in (22) to obtain

$$
9 q\left(\frac{y}{3}\right)+2 q(x)+q(y)=4 q\left(\frac{x+y}{2}\right)+4 q\left(\frac{y-x}{2}\right) .
$$

Taking $y=0$ in (23) one has

$$
q(x)=4 q\left(\frac{x}{2}\right)
$$

whence the relation

$$
q(2 x)=4 q(x)
$$

follows. Further putting $x=0$ in (23) leads to the relation

$$
9 q\left(\frac{y}{3}\right)+q(y)=8 q\left(\frac{y}{2}\right) \text {. }
$$

whence by $(24)$

$$
q(3 y)=9 q(y)
$$

Finally, with (24) and (25), equality (23) becomes

$$
2 q(x)+2 q(y)=q(x+y)+q(x-y),
$$

i.e., $q$ is a quadratic function.

At the end notice that

$$
\begin{aligned}
& a(x)+q(x)=\frac{1}{2}[f(x), f(-x)]+\frac{1}{2}[f(x)+f(-x), 2 f(0)] \\
& =\frac{1}{2}[2 f(x)+f(-x), f(-x)+2 f(0)]=\frac{1}{2}[2 f(x), 2 f(0)] \\
& =[f(x), f(0)]=F(x) .
\end{aligned}
$$

Theorem 4. A function $f: M \rightarrow S$ is a solution of (15) if and only if $f$ satisfies the system of the functional equations

$$
\begin{aligned}
& f(x+y)+f(-x)+f(-y)=f(-x-y)+f(x)+f(y) \\
& 2 f(0)+f(x+y)+f(x-y)=2 f(x)+f(y)+f(-y) .
\end{aligned}
$$

Proof. Assume that $f$ is a solution of (15). Theorem 3 says that

$$
a(x)=\frac{1}{2}[f(x), f(-x)]
$$

is an additive function what means $a(x+y)=a(x)+a(y)$ for $x, y \in M$, or

$$
[f(x+y), f(-x-y)]=[f(x), f(-x)]+[f(y), f(-y)] .
$$


(27) is an immediate consequence of (29). Similarly

$$
q(x)=\frac{1}{2}[f(x)+f(-x), 2 f(0)]
$$

is quadratic. Thus $q$ satisfies equation (26). Consequently

$$
\begin{aligned}
& {[f(x)+f(-x), 2 f(0)]+[f(y)+f(-y), 2 f(0)]} \\
& =\frac{1}{2}[f(x+y)+f(-x-y), 2 f(0)]+\frac{1}{2}[f(x-y)+f(-x+y), 2 f(0)] .
\end{aligned}
$$

The last equality may be rewritten as

$$
\begin{aligned}
& f(x+y)+f(-x-y)+f(x-y)+f(-x+y)+4 f(0) \\
& =2 f(x)+2 f(-x)+2 f(y)+2 f(-y)
\end{aligned}
$$

or

$$
\begin{aligned}
& f(x+y)+f(x-y)+f(-x-y)+f(x)+f(y)+f(-x+y) \\
& +f(x)+f(-y)+4 f(0)=4 f(x)+2 f(-x)+3 f(y)+3 f(-y) .
\end{aligned}
$$

From (27) the left-hand side of the above relation may be rewritten as $f(x+y)+f(x-y)+f(x+y)+f(-x)+f(-y)+f(x-y)+f(-x)+f(y)+4 f(0)$. Cancelling $2 f(-x)+f(y)+f(-y)$ we obtain

$$
2 f(x+y)+2 f(x-y)+4 f(0)=4 f(x)+2 f(y)+2 f(-y),
$$

whence (28) follows.

Conversely, if $f: M \rightarrow S$ is a solution of system (27)-(28), then $a$ given by formula

$$
a(x)=\frac{1}{2}[f(x), f(-x)]
$$

is an additive function by (27) and

$$
q(x)=\frac{1}{2}[f(x)+f(-x), 2 f(0)]
$$

is a quadratic one by (28) and (27). Thus both of them are solutions of equation (17). Consequently their sum

$$
a(x)+q(x)=F(x)=[f(x), f(0)]
$$

is also a solution of this equation, whence it follows that $f$ satisfies (15).

Remark 1. If $f$ is a solution of $(15)$, then $g(y)=(1 / 2)(f(y)+f(-y))$ is an even solution of this equation. From Theorem 2, $g(y)=g(0)+q(y)$, where $q$ is a quadratic function. Thus (28) may be rewritten as follows

$$
f(x+y)+f(x-y)=2 f(x)+2 q(y) .
$$


Remark 2. We are not able to solve the system of equations (27)-(28) in the class of functions $f: M \rightarrow S$. It is known that the only solutions of equation (15) in the class of functions $f: M \rightarrow Y$, where $Y$ is a real vector space, are of the form

$$
f(x)=b+a(x)+q(x),
$$

where $b \in Y, a$ is additive and $q$ is quadratic (cf. [3]). Thus with respect to Theorem 4 all solutions of the system of (27)-(28) are of form (30).

4. Let $X$ be a real Banach space and let $\operatorname{clb}(X)$ denote the hyperspace of all non-empty convex closed bounded subset of $X$. It is clear that $\operatorname{clb}(X)$ is an abstract convex cone with the addition given by

$$
A \stackrel{*}{+} B=\operatorname{cl}(A+B),
$$

where $\mathrm{cl} A$ denotes the closure of the set $A$, and with the multiplication $\lambda A$ by non-negative numbers $\lambda$. The identity element of $\operatorname{clb}(X)$ is the singleton $\{0\}$. In this convex cone the cancellation law holds true. This is a consequence of a generalization (cf. [7], also [1, Theorem II-17, p. 48]) of the Rådström's lemma (cf. [4]). The convex cone $\operatorname{clb}(X)$ may be endowed with the Hausdorff metric

$$
h(A, B)=\inf \{t>0: A \subset B+t K, B+t K\},
$$

where $K$ is the closed unit ball in $X$. The metric $h$ is translation invariant (cf. [2]), positively homogeneous and complete (cf. [1]) in $\mathrm{clb}(X)$ ). Consequently all the obtained results may be transfered to the set-valued case. For example we can derive the following result from Theorem 1.

Theorem 5. Assume that $b \geq 1$. If $F: M \rightarrow \operatorname{clb}(X)$ is a solution of the functional equation

$$
F\left(\frac{x+y}{2}\right)=\frac{1}{2}(F(x) \stackrel{*}{+} F(y)), \quad x, y \in M,
$$

then $F$ is a solution of the functional equation

$$
\begin{aligned}
& 3(b-1) F\left(\frac{x+y+z}{3}\right) \stackrel{*}{+} F(x) \stackrel{*}{+} F(y) \stackrel{*}{+} F(z) \\
& =b\left[F\left(\frac{x+y}{2}\right)^{*}+F\left(\frac{y+z}{2}\right) \stackrel{*}{+} F\left(\frac{z+x}{2}\right)\right] .
\end{aligned}
$$

Conversely, if $b \geq 1, b \neq 4$ and $F: M \rightarrow \operatorname{clb}(X)$ is a solution of (32), then $F$ satisfies the Jensen functional equation (31). 


\section{References}

[1] Castaing, C., Valadier, M., Convex Analysis and Measurable Multifunctions, Lecture Notes in Math. 580, Springer-Verlag, Berlin, Heidelberg, New York, 1977.

[2] De Blasi, F.S., On differentiability of multifunctions, Pacific J. Math. 66 (1976), 67-81.

[3] Lee, Y. W., On the stability of a quadratic Jensen type functional equation, J. Math. Anal. Appl. 270 (2002), 590-601.

[4] Rådström, H., An embedding theorem for spaces of convex sets, Proc. Amer. Math. Soc. 3 (1952), 165-169.

[5] Smajdor, W., On Jensen and Pexider functional equations, Opuscula Math. 14 (1994), 169-178.

[6] Smajdor, W., Note on a Jensen type functional equation, Publ. Math. Debrecen 63(4) (2003), 703-714.

[7] Urbański, R., A generalization of Minkowski-Rådström-Hörmander theorem, Bull. Acad. Polon. Sci. Sér. Sci. Math. Astronom. Phys. 24(9) (1976), 709-715.

[8] Trif, T., Hyers-Ulam-Rassias stability of a Jensen type functional equation, J. Math. Anal. Appl. 250 (2000), 579-588.

WiLHELMina SMAJDOR

Silesian University of TeChNOLOGY

KASZUBSKA 23

44-100 GLiwice

POLAND

E-MAIL: W.SMAJDOR@POLSL.PL 